Received: 09.08.2019

Revised: 15.10 .2019

Accepted: 25.10 .2019

DOI: $10.17804 / 2410-9908.2019 .5 .023-039$

\title{
AN EXACT SOLUTION TO THE DESCRIPTION OF A UNIDIRECTIONAL MARAN- GONI FLOW OF A VISCOUS INCOMPRESSIBLE FLUID WITH THE NAVIER BOUNDARY CONDITION. VELOCITY FIELD INVESTIGATION
}

\author{
N. V. Burmasheva ${ }^{\text {a** }}$ and E. Yu. Prosviryakov ${ }^{\text {b) }}$ \\ Institute of Engineering Science, Ural Branch of the Russian Academy of Sciences, \\ 34 Komsomolskaya St., Ekaterinburg, 620049, Russian Federation \\ a) iD https://orcid.org/0000-0003-4711-1894 nat_burm@mail.ru; \\ b) iD https://orcid.org/0000-0002-2349-7801 《 evgen_pros@mail.ru \\ *Corresponding author. E-mail: nat_burm@mail.ru \\ Address for correspondence: 34 Komsomolskaya St., Ekaterinburg, 620049, Russian Federation \\ Tel.: +7 (343) 37535 76; fax: +7 (343) 3745330
}

The article considers the unidirectional flow of a viscous incompressible fluid in an infinite horizontal layer of a given thickness, which is induced by the thermocapillary effect specified at the upper boundary of the layer and taking into account the condition of fluid slipping at the lower boundary. An exact solution to the Oberbeck-Boussinesq equation system is obtained. A detailed analysis of the velocity field was carried out for various slip length values. It is shown that counterflows may occur in a fluid. Their number is analyzed, the conditions for the appearance of velocity field stratification are written.

Keywords: layered flow, Oberbeck-Boussinesq equation system, exact solution, unidirectional flow, counterflows, Marangoni convection, Navier condition.

\section{References}

1. Marangoni C. Sull espansione delle goccie di un liquido galleggiante sulla superficie di altro liquid, Pavia, Tipografia dei fratelli Fusi, 1865.

2. Goldstein S.V. Modern Developments in Fluid Mechanics, Oxford, Oxford Univ. Press, 1938.

3. Prandtl L., Tietjens O. Hydro- und aeromechanic (2 vols.), Berlin, Verlag von Julius Springer, 1931. DOI: 10.1017/S0368393100115366.

4. Borzenko E.I., Shrager G.R. The structure of viscoplastic fluid flow during filling of a circular pipe/plane channel. Computational continuum mechanics, 2019, vol. 12, no. 2, pp. 129-136. DOI: 10.7242/1999-6691/2019.12.2.11. (In Russian).

5. Neto C., Evans D., Bonaccurso E. Boundary slip in Newtonian liquids: a review of experimental studies. Reports on Progress in Physics, 2005, vol. 68, no. 12, pp. 2859-2897. DOI: $10.1088 / 0034-4885 / 68 / 12 / R 05$.

6. Pelenko V.V., Aret V.A., Gusev B.K., Pelenko F.V. The flow of viscoplastic nonlinear media with boundary slip. In: The Bulletin of KrasGAU: Interuniversity Collection of Scientific Papers, Krasnoyarsk, KrasGAU, 2008, no. 2, pp. 54-57. (In Russian).

7. Navier C.L.M.H. M'emoire sur les lois du mouvement des fluids. M'em. Acad. Sci. Inst. de France, 1823, vol. 2, no. 6. pp. 389-440.

8. Lauga E., Brenner M., Stone H. Microfluidics: The No-Slip Boundary Condition. In: C. Tropea, A.L. Yarin, J.F. Foss, ed., Springer Handbook of Experimental Fluid Mechanics, 
Springer, Berlin, Heidelberg, Springer Handbooks, 2007.

9. Hoffmann J., Johnson C. Computational Turbulent Incompressible Flow, Heidelberg, Berlin, Springer-Verlag, 2007, 397 p.

10. Borzenko E.I., Diakova O.A., Shrager G.R. Studying the slip phenomenon for a viscous fluid flow in a curved channel. Tomsk State University Journal of Mathematics and Mechanics, 2014, no. 2 (28), pp. 35-44. (In Russian).

11. Privalova V.V., Prosviryakov E.Yu. Nonlinear isobaric flow of a viscous incompressible fluid in a thin layer with permeable boundaries. Computational continuum mechanics, 2019, vol. 12, no. 2, pp. 230-242. DOI: 10.7242/1999-6691/2019.12.2.20. (In Russian).

12. Gershuni G.Z., Zhukhovitskii E.M. Convective Stability of Incompressible Fluids, Israel Program for Scientific Translations, Jerusalem, Keter Publishing House, 1976, 330 pp.

13. Ostroumov G.A. Free convection under the condition of the internal problem, Washington, NACA Technical Memorandum 1407, National Advisory Committee for Aeronautics, 1958.

14. Birikh R.V. Thermocapillary convection in a horizontal layer of liquid. J. Appl. Mech. Tech. Phys., 1966, vol. 7, no. 3, p. 43.

15. Sidorov A.F. Two classes of solutions of the fluid and gas mechanics equations and their connection to traveling wave theory. Journal of Applied Mechanics and Technical Physics, 1989, vol. 30, iss. 2, pp 197-203, 1989, no. 2, pp. 34-40. (In Russian).

16. Andreev V.K., Gaponenko Ya.A., Goncharova O.N., Pukhnachev V.V. Mathematical Models of Convection, Berlin-Boston, De Gryuter Publ., 2012. xv++ 417 p.

17. Aristov S.N., Prosviryakov E.Yu. A new class of exact solutions for three-dimensional thermal diffusion equations. Theor. Found. Chem. Eng., 2016, vol. 50, no. 3, pp. 286-293. DOI: $10.1134 / \mathrm{S} 0040579516030027$.

18. Aristov S.N., Frik P.G. Nonlinear effects of the Ekman layer on the dynamics of largescale eddies in shallow water. J. Appl. Mech. Tech. Phys., 1991, vol. 32, no. 2, pp. 189-194. DOI: $10.1007 / \mathrm{BF} 00858033$.

19. Ingel L.Kh., Aristov S.N. The class of exact solutions of nonlinear problems on thermal circulation associated with volumetric heat release in the atmosphere. Tr. In-ta Eksperim. Meteorol., 1996, no. 27 (162), pp. 142-157. (In Russian).

20. Pukhnachev V.V. Non-stationary analogues of the Birikh solution. Izvestiya AltGU, 2011, no. 1-2, pp. 62-69. (In Russian).

21. Aristov S.N., Prosviryakov E.Yu. On laminar flows of planar free convection. Russian Journal of Nonlinear Dynamics, 2013, vol. 9, no. 4, pp. 651-657. DOI: 10.20537/nd1304004. (In Russian).

22. Andreev V.K. Resheniya Birikha uravneniy konvektsii $i$ nekotorye ego obobshcheniya: preprint [Birikh Solutions of Convection Equations and Some of its Generalizations: preprint]. Krasnoyarsk, 2010, № 1-10. (In Russian).

23. Pukhnachev V.V. Group-theoretical methods in the convection problems. In: Application of Mathematics in Technical and Natural Sciences, M.D. Todorov and C.I. Christov, eds., American Institute of Physics, CP 1404, Melwille, NY, 2011, pp. 31-42.

24. Burmasheva N.V., Prosviryakov E.Yu. Convective layered flows of a vertically whirling viscous incompressible fluid. Velocity field investigation. Vestn. Samar. Gos. Tekhn. Univ., Ser. Fiz.-Mat. Nauki [J. Samara State Tech. Univ., Ser. Phys. Math. Sci.], 2019, vol. 23, no. 2, pp. 341-360. DOI: 10.14498/vsgtu1670.

25. Aristov S.N., Prosviryakov E.Yu., Spevak L.F. Unsteady-state Bйnard-Marangoni convection in layered viscous incompressible flows. Theor. Found. Chem. Eng., 2016, vol. 50, no. 2, pp. 132-141. DOI: 10.1134/S0040579516020019.

26. Burmasheva N.V., Prosviryakov E.Yu. Temperature field investigation in layered flows of a vertically swirling viscous incompressible fluid under two thermocapillar forces 
at a free boundary. Diagnostics, Resource and Mechanics of materials and structures, 2019, iss. 1, pp. 6-42. DOI: 10.17804/2410-9908.2019.1.006-042. Available at: http://dreamjournal.org/DREAM_Issue_1_2019_Burmasheva_N.V._et_al._006_042.pdf

27. Gorshkov A.V., Prosviryakov E.Yu. Layered Benard-Marangoni convection during heat transfer according to the Newton's law of cooling. Comp. Research and Modeling, 2016, vol. 8, no. 6, pp. 927-940. (In Russian).

28. Goncharova O.N., Kabov O.A. Gravitational-thermocapillary convection of fluid in the horizontal layer in co-current gas flow. Dokl. Phys., 2009, vol. 54, no. 5, pp. 242-247. DOI: $10.1134 / \mathrm{S} 1028335809050061$.

29. Bekezhanova V.B. Convective instability of Marangoni-Poiseuille flow under a longitudinal temperature gradient. Journal of Applied Mechanics and Technical Physics, 2011, vol. 52, no. 1, pp. 74-81. DOI: 10.1134/S0021894411010111.

30. Gordeeva V.Y., Lyushnin A.V. Influence of the thermocapillary effect on the dynamics and stability of motion of a thin evaporating film. Technical Physics, 2013, vol. 58, no. 3, pp. 351-357. DOI: $10.1134 /$ S1063784213030092.

31. Aktershev S.P. Thermocapillary effect and periodic structures on the surface of a heated viscous liquid film. In: Proceedings of the Institute of Mechanics of Ural Branch of RAS, 2007, no. 5, pp. 79-84. DOI: 10.21662/uim2007.1.005. (In Russian).

32. Schlunder E.U. Heat Exchanger Design Handbook, Hemisphere Publishing Corporation, 1983.

33. Aristov S.N., Prosviryakov E.Yu. Nonuniform convective Couette flow. Fluid Dynamics, 2016, vol. 51, no. 5, pp. 581-587. DOI: 10.1134/S001546281605001X.

34. Prosviryakov E.Yu. A new class of exact solutions of the Navier - Stokes equations with a power-law dependence of velocities on two spatial coordinates. Theoretical Foundations of Chemical Engineering, 2019, vol. 53, no. 1, pp. 112-120. DOI: 10.1134/S0040357118060118. (In Russian).

35. Burmasheva N.V., Prosviryakov E.Yu. A large-scale layered stationary convection of a incompressible viscous fluid under the action of shear stresses at the upper boundary. Velocity field investigation. Vestn. Samar. Gos. Tekhn. Univ., Ser. Fiz.-Mat. Nauki [J. Samara State Tech. Univ., Ser. Phys. Math. Sci.]. 2017, vol. 21, no. 1, pp. 180-196. DOI: 10.14498/vsgtu1527. (In Russian).

36. Knutova N.S., Shvarts K.G. A study of behavior and stability of an advective thermocapillary flow in a weakly rotating liquid layer under microgravity. Fluid Dyn., 2015, vol. 50, no. 3, pp. 340-350. DOI: 10.1134/S0015462815030047.

37. Couette M. Études sur le frottement des liquids. Ann. Chim. Phys. Ser. 6, 1890, vol. 21, pp. 433-510. 
Подана в журнал: 09.08.2019

УДК 539

DOI: $10.17804 / 2410-9908.2019 .5 .023-039$

\title{
ТОЧНОЕ РЕШЕНИЕ ДЛЯ ОПИСАНИЯ ОДНОНАПРАВЛЕННОГО ТЕЧЕНИЯ МАРАНГОНИ ВЯЗКОЙ НЕСЖИМАЕМОЙ ЖИДКОСТИ С ГРАНИЧНЫМ УСЛОВИЕМ НАВЬЕ. ИССЛЕДОВАНИЕ ПОЛЯ СКОРОСТИ
}

\author{
Н. В. Бурмашева ${ }^{\text {a)* }}$, Е. Ю. Просвиряков ${ }^{\text {() }}$ \\ Институт машиноведения УрО РАН, \\ ул. Комсомольская, 34, Екатеринбург, Российская Федерация \\ a) (iD https://orcid.org/0000-0003-4711-1894 nat_burm@mail.ru; \\ б) iD https://orcid.org/0000-0002-2349-7801 @ evgen_pros@mail.ru \\ *Ответственный автор. Электронная почта: nat_burm@mail.ru \\ Адрес для переписки: ул. Комсомольская, 34, Екатеринбург, Россия \\ Тел.: +7 (343) 375-35-76; факс: 374-53-30
}

В статье рассматривается однонаправленное течение вязкой несжимаемой жидкости в бесконечном горизонтальном слое заданной толщины, индуцированное заданным на верхней границе слоя термокапиллярным эффектом и учетом на нижней границе условия проскальзывания жидкости. Получено точное решение системы уравнений Обербека-Буссинеска. Проведен подробный анализ поля скорости для различных значений длины проскальзывания. Показано, что в жидкости могут возникать противотечения. Проанализировано их число, выписаны условия появления стратификации поля скорости.

Ключевые слова: слоистое течение, система уравнений Обербека-Буссинеска, точное решение, однонаправленное течение, противотечения, конвекция Марангони, условие Навье.

\section{1. Введение}

Основой многих технологических процессов является движение жидкости в различного рода системах. Причем особенности переноса энергии, импульса и вещества в ходе этих процессов зависят от множества факторов, к числу которых относятся задаваемые на границах области течения краевые условия. Тип краевого условия определяется видом контактирующих поверхностей. На границе раздела двух сред достаточно часто используется условие, описывающее термокапиллярный эффект Марангони-Гиббса [1]. На границе жидкости с твердой поверхностью наиболее распространено использование условия прилипания [2-4]. Однако экспериментальные работы [5] показывают, что это условие выполняется не всегда. Тогда в качестве альтернативы берется условие проскальзывания. Существует несколько типов условий проскальзывания [6-11]. Выбор конкретного вида граничного условия оказывает существенное влияние на решение краевой задачи.

Изучение конвективных движений в жидкости основывается на анализе свойств точных решений уравнений тепловой конвекции [12]. В работах [13-31] построены обобщенные классы точных решений, в том числе и для конвективных термокапиллярных течений [25-31]. Предложенные точные решения описывают неодномерные течения жидкости. Тем не менее в некоторых случаях при проектировании аппаратов, использующих жидкость с низкой температурой плавления или высокой вязкостью, сама технология диктует применение однонаправленного течения теплоносителей. Однонаправленное течение обеспечивает наиболее однородную температуру стенок между входом и выходом аппарата, сводя тем самым к минимуму температурные напряжения. [32]. 
В предложенной работе построено точное решение, описывающее однонаправленное течение Марангони вязкой несжимаемой жидкости с учетом условия проскальзывания Навье [7] на твердой границе горизонтального бесконечного слоя. Построенное решение для поля температуры и поля давления оказывается двумерным по координатам. Основное внимание в статье уделено анализу поля скоростей течения. Показано, что решение допускает появление расслоений. Выписано условие на граничные параметры, удовлетворение которого означает наличие точек расслоения поля скорости.

\section{2. Постановка задачи и методы решения}

Рассматривается установившееся конвективное слоистое течение вязкой несжимаемой жидкости в плоском горизонтальном бесконечном слое толщины $h$. Действующее на жидкость поле силы тяжести характеризуется ускорением свободного падения $\boldsymbol{g}$, направленного вертикально вниз. Полагаем, что справедливо приближение Обербека-Буссинеска о зависимости плотности жидкости $\rho$ от ее температуры $T \rho=\rho_{0}(1-\beta T)$, где $\beta$ - коэффициент теплового расширения жидкости, $\rho_{0}$ - среднее значение плотности [12].

Для описания конвективного течения вязкой несжимаемой жидкости введем систему координат таким образом, чтобы ось $O z$ была направлена вертикально вверх, а оси $O x, O y$ лежали в горизонтальной плоскости.

Полная система уравнений тепловой конвекции, используемая для описания слоистых течений (компонента $V_{z}$ вектора скорости $\boldsymbol{V}$ полагается равной нулю) вязкой несжимаемой жидкости, имеет вид [12]:

$$
\begin{gathered}
V_{x} \frac{\partial V_{x}}{\partial x}+V_{y} \frac{\partial V_{x}}{\partial y}=v \Delta V_{x}-\frac{\partial P}{\partial x} \\
V_{x} \frac{\partial V_{y}}{\partial x}+V_{y} \frac{\partial V_{y}}{\partial y}=v \Delta V_{y}-\frac{\partial P}{\partial y} \\
\frac{\partial P}{\partial z}=g \beta \Delta T \\
V_{x} \frac{\partial T}{\partial x}+V_{y} \frac{\partial T}{\partial y}=\chi \Delta T \\
\frac{\partial V_{x}}{\partial x}+\frac{\partial V_{y}}{\partial y}=0
\end{gathered}
$$

В системе (1) приняты обозначения: $P(x, y, z)$ - нормированное на плотность отклонение давления от гидростатического; $T(x, y, z)$ - отклонение температуры от отсчетного значения $v ; \chi-$ кинематическая (молекулярная) вязкость жидкости и ее температуропроводность; $\Delta=\frac{\partial^{2}}{\partial x^{2}}+\frac{\partial^{2}}{\partial y^{2}}+\frac{\partial^{2}}{\partial z^{2}}-$ оператор Лапласа. Первые три уравнения системы (1) есть запись векторного уравнения Навье-Стокса в проекциях на координатные оси, четвертое уравнение - уравнение теплопроводности, последнее - уравнение несжимаемости.

В случае рассмотрения однонаправленных течений вдоль оси $O x$ (рис. 1) полагаем скорость $V_{y}=0$. Тогда система (1) значительно упрощается: 


$$
\begin{gathered}
V_{x} \frac{\partial V_{x}}{\partial x}=-\frac{\partial P}{\partial x}+v\left(\frac{\partial^{2} V_{x}}{\partial x^{2}}+\frac{\partial^{2} V_{x}}{\partial y^{2}}+\frac{\partial^{2} V_{x}}{\partial z^{2}}\right) \\
\frac{\partial P}{\partial y}=0 ; \frac{\partial P}{\partial z}=g \beta T \\
V_{x} \frac{\partial T}{\partial x}=\chi\left(\frac{\partial^{2} T}{\partial x^{2}}+\frac{\partial^{2} T}{\partial y^{2}}+\frac{\partial^{2} T}{\partial z^{2}}\right) \\
\frac{\partial V_{x}}{\partial x}=0
\end{gathered}
$$

Стоит отметить, что системы (1) и (2) являются переопределенными, так как в них число уравнений превосходит число неизвестных функций, в роли которых выступают компоненты вектора скорости, давление и температура. Разрешимость системы вида (1) и точные решения для нее обсуждались в [17, 21, 24-27, 33-35].

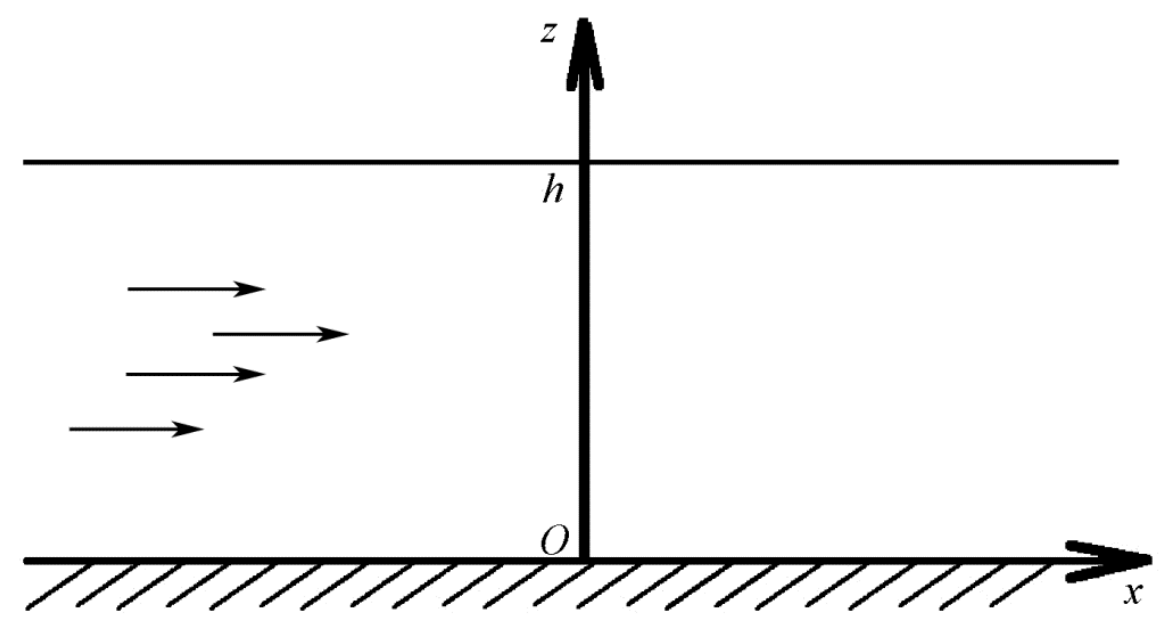

Рис. 1. Геометрия слоя

Из последнего уравнения системы (2) следует, что скорость жидкости зависит только от двух координат:

$$
V_{x}=V_{x}(y, z)
$$

Принимая во внимание соотношение (3), систему (2) можно переписать в следующем виде:

$$
\begin{gathered}
\frac{\partial P}{\partial x}=v\left(\frac{\partial^{2} V_{x}}{\partial y^{2}}+\frac{\partial^{2} V_{x}}{\partial z^{2}}\right) ; \frac{\partial P}{\partial z}=g \beta T \\
V_{x} \frac{\partial T}{\partial x}=\chi\left(\frac{\partial^{2} T}{\partial x^{2}}+\frac{\partial^{2} T}{\partial y^{2}}+\frac{\partial^{2} T}{\partial z^{2}}\right)
\end{gathered}
$$

при этом $P=P(x, z)$. 
Далее будем рассматривать течения вязкой несжимаемой жидкости, у которых профиль скорости зависит только от одной (поперечной) координаты $[35,36]$ :

$$
V_{x}=U(z)
$$

Формула (5) для течений жидкости при постоянной температуре описывает профиль Куэтта [37], а для конвективных течений - профиль Остроумова-Бириха [13-14].

Подстановка выражения (5) в систему (4) приводит к системе дифференциальных уравнений в частных производных:

$$
\begin{gathered}
\frac{\partial P}{\partial x}=v \frac{\partial^{2} U}{\partial z^{2}} \\
\frac{\partial P}{\partial z}=g \beta T ; \\
U \frac{\partial T}{\partial x}=\chi\left(\frac{\partial^{2} T}{\partial x^{2}}+\frac{\partial^{2} T}{\partial y^{2}}+\frac{\partial^{2} T}{\partial z^{2}}\right) .
\end{gathered}
$$

Кроме того, использование семейства решений (5) в силу системы (6) позволяет определить структуру решения для гидродинамических полей температуры $T$ и давления $P$. Несложно убедиться, что поле давления и поле температуры оказываются линейными формами введенных выше координат:

$$
T=T_{0}(z)+T_{1}(z) x ; P=P_{0}(z)+P_{1}(z) x .
$$

Подставляя соотношения (5) и (7) в систему (6), получим систему обыкновенных дифференциальных уравнений относительно переменной $z$ (дифференцирование по этой переменной обозначено штрихом):

$$
\begin{gathered}
T_{1}^{\prime \prime}=0 ; P_{1}^{\prime}=g \beta T_{1} ; v U^{\prime \prime}=P_{1} ; \\
\chi T_{0}^{\prime \prime}=U T_{1}, P_{0}^{\prime}=g \beta T_{0} .
\end{gathered}
$$

Общее решение системы (8) имеет вид:

$$
\begin{gathered}
T_{1}=C_{1} z+C_{2} ; P_{1}=\frac{1}{2} g z^{2} \beta C_{1}+g z \beta C_{2}+C_{3} ; \\
U=\frac{g z^{4} \beta C_{1}}{24 v}+\frac{g z^{3} \beta C_{2}}{6 v}+\frac{z^{2} C_{3}}{2 v}+z C_{4}+C_{5} ; \\
T_{0}=\frac{1}{7} g z^{7} \beta C_{1}^{2}+g z^{6} \beta C_{1} C_{2}+z^{5}\left[\frac{6}{5} g \beta C_{2}^{2}+\frac{18}{5} C_{1} C_{3}\right]+6 z^{4}\left[C_{2} C_{3}+2 v C_{1} C_{4}\right]+ \\
+24 z^{3} v\left(C_{2} C_{4}+C_{1} C_{5}\right)+72 z^{2} v C_{2} C_{5}+144 z v \chi C_{6}+C_{7} ; \\
P_{0}=\frac{g^{2} z^{8} \beta^{2} C_{1}^{2}}{8064 v \chi}+\frac{g^{2} z^{7} \beta^{2} C_{1} C_{2}}{1008 v \chi}+\frac{g z^{6} \beta\left[g \beta C_{2}^{2}+3 C_{1} C_{3}\right]}{720 v \chi}+\frac{g z^{5} \beta\left[C_{2} C_{3}+2 v C_{1} C_{4}\right]}{120 v \chi}+ \\
+\frac{g z^{4} \beta\left(C_{2} C_{4}+C_{1} C_{5}\right)}{24 \chi}+\frac{g z^{3} \beta C_{2} C_{5}}{6 \chi}+\frac{1}{2} g z^{2} \beta C_{6}+g z \beta C_{7}+C_{8} .
\end{gathered}
$$


Выражения (9) содержат восемь неизвестных констант интегрирования $\left(C_{i}, i=\overline{1,8}\right)$, следовательно, для их нахождения необходимо определить восемь краевых условий.

Будем полагать, что задана температура нагрева верхней границы $z=h$, а температура на нижней границе $z=0$ принята за отсчетный уровень:

$$
T(0)=0 ; T(h)=A x
$$

Условия (10) в силу представления (7) равносильны следующим условиям:

$$
T_{0}(0)=T_{1}(0)=0 ; T_{0}(h)=0 ; T_{1}(h)=A .
$$

Также полагаем, что на нижней границе выполняется условие скольжения Навье [7], а на верхней - задано постоянное давление $S_{0}$, соответствующее атмосферному давлению, и поле касательных напряжений, вызванное термокапиллярным эффектом [1]:

$$
\left.\alpha \frac{\partial V_{x}}{\partial \mathbf{n}}\right|_{z=0}=V_{x}(0) ; P(h)=S_{0} ;\left.\eta \frac{\partial V_{x}}{\partial z}\right|_{z=h}=\left.\frac{\partial T}{\partial x}\right|_{z=h} .
$$

Здесь $\mathbf{n}$ - вектор нормали, направленный от нижней границы слоя жидкости внутрь слоя жидкости [7]. С помощью класса (5) и (7) граничные условия (12) записываются в виде:

$$
\left.\alpha \frac{\partial U}{\partial z}\right|_{z=0}=U(0) ; P_{0}(h)=S_{0} ; P_{1}(h)=0 ;\left.\eta \frac{\partial U}{\partial z}\right|_{z=h}=-\sigma T_{1}(h) .
$$

Здесь $\sigma$ и $\eta-$ коэффициенты температурного поверхностного натяжения и динамической вязкости соответственно; $\alpha$ - длина проскальзывания.

\section{3. Результаты и обсуждение}

\section{1. Точное решение краевой задачи}

Решение краевой задачи (8), (11), (13) является полиномиальным:

$$
\begin{gathered}
T_{1}=\frac{A z}{h} ; P_{1}=\frac{A g \beta}{2 h}\left(z^{2}-h^{2}\right) \\
U=A\left\{\frac{g \beta}{24 v h}\left[z^{4}-6 h^{2} z^{2}+8 h^{3}(z+\alpha)\right]-\frac{\sigma}{\eta}(z+\alpha)\right\} \\
T_{0}=A^{2}\left\{\frac{\sigma}{12 h \chi \eta}(h-z) z\left[h^{2}+(h+z)(z+2 \alpha)\right]+\right. \\
\left.+\frac{g \beta}{5040 h^{2} v \chi}\left[5 z^{7}-63 h^{2} z^{5}+140 h^{3} z^{4}+280 h^{3} z^{3} \alpha-2 h^{5} z(41 h+140 \alpha)\right]\right\} \\
P_{0}=S_{0}-\frac{A^{2} g \beta \sigma}{120 h \eta \chi}(z-h)^{2}\left(3 h^{3}+2 h z(2 z+5 \alpha)+z^{2}(2 z+5 \alpha)+h^{2}(6 z+5 \alpha)\right)+ \\
+\frac{A^{2} g^{2} \beta^{2}}{40320 h^{2} v \chi}(z-h)^{2}\left[183 h^{6}-69 h^{2} z^{4}+10 h z^{5}+5 z^{6}+\right.
\end{gathered}
$$




$$
\left.+h^{4} z(221 z+1120 \alpha)+h^{5}(366 z+560 \alpha)+4 h^{3} z^{2}(19 z+140 \alpha)\right] .
$$

Легко видеть, что наименьшую степень имеет полином, описывающий свойства продольного градиента температуры $T_{1}$, а наибольшую - полином, отвечающий фоновому давлению $P_{0}$.

Заметим, что точное решение (14) обладает тем свойством, что нулевые точки всех компонент полей (за исключением фонового давления $P_{0}$ ) не зависят от величины значения горизонтального температурного градиента $A$. Влияние этого градиента на фоновое давление $P_{0}$ можно нивелировать произволом выбора значения $S_{0}$.

Далее в статье будем обсуждать только поле скорости течения.

\section{2. Исследование скорости течения в случае прилипания жидкости и граничного условия для проскальзывания жидкости}

Проанализируем поле скорости, представляемое компонентой $U$. Очевидно, что в случае теплоизолированных границ $(A=0)$ жидкость будет находиться в покое относительной выбранной системы координатных осей. Если температурный градиент $A$ отличен от нуля, то скорость $U$ определяется нелинейным взаимодействием двух потоков - термогравитационного $\frac{A g \beta}{24 v h}\left[z^{4}-6 h^{2} z^{2}+8 h^{3}(z+\alpha)\right]$ и термокапиллярного $\frac{A \sigma}{\eta}(z+\alpha)$. Вклад каждого потока зависит от величин коэффициентов $\frac{A g \beta}{24 v h}$ и $\frac{A \sigma}{\eta}$. Представим тогда выражение скорости (14) в следующем виде:

$$
U=A h\left\{\frac{g \beta h^{2}}{24 v}\left[Z^{4}-6 Z^{2}+8 Z\right]-\frac{\sigma}{\eta} Z+\frac{a}{\delta}\left(\frac{g \beta h^{2}}{24 v}-\frac{\sigma}{\eta}\right)\right\} .
$$

В формуле (15) введены обозначения: $Z=\frac{z}{h} \in[0,1]$ - безразмерная координата, $a=\frac{\alpha}{l}$ $; \delta=\frac{h}{l} ; l-$ характерный горизонтальный размер рассматриваемого слоя жидкости.

Отметим, что полиномы $f_{1}=Z^{4}-6 Z^{2}+8 Z$ и $f_{2}=Z$ являются строго монотонными на отрезке $[0,1]$ (рис. 2), линейно независимыми и принимают положительные значения на $(0,1)$. Поэтому в виду неотрицательности параметра $\frac{a}{\delta}$ по отдельности ни термогравитационный, ни термокапиллярный потоки не могут привести к появлению застойной точки скорости $U$.

Заметим, что если $a=0$, то условие Навье на нижней границе слоя вырождается в условие прилипания. В этом случае скорость (15) может иметь застойную (нулевую точку) только для ньютоновских жидкостей. Действительно, если жидкость аномальная $(\sigma<0)$, то коэффициент $-\frac{A \sigma}{\eta}>0$, значит скорость $U$ описывается суммой двух неотрицательных строго возрастающих функций, обращающихся в нуль только на нижней границе слоя жидкости. Сумма таких функций также будет неотрицательной строго возрастающей функцией, 
следовательно, всюду внутри слоя скорость $U$ будет отлична от нуля, при этом ее направление будет определяться только знаком продольного градиента температуры $A$.

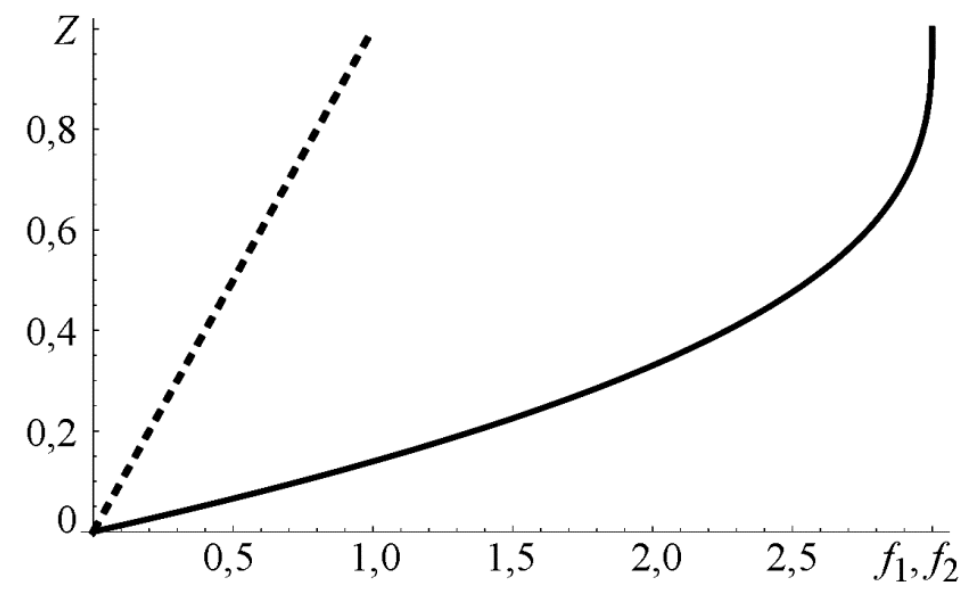

Рис. 2. Профили вспомогательных функций $f_{1}$ (сплошная линия) и $f_{2}$ (пунктирная линия)

Вернемся к случаю положительной безразмерной длины скольжения ( $a>0)$. Если некоторая точка $Z_{1}$ является нулем многочлена (15) на отрезке $[0,1]$, то выражение

$$
0=U\left(Z_{1}\right)=A h\left\{\frac{g \beta h^{2}}{24 v}\left[Z_{1}^{4}-6 Z_{1}^{2}+8 Z_{1}\right]-\frac{\sigma}{\eta} Z_{1}+\frac{a}{\delta}\left(\frac{g \beta h^{2}}{24 v}-\frac{\sigma}{\eta}\right)\right\}
$$

можно рассматривать как однородное линейное уравнение относительно коэффициентов $\frac{g \beta h^{2}}{24 v}, \frac{\sigma}{\eta}$ и $\frac{a}{\delta}\left(\frac{g \beta h^{2}}{24 v}-\frac{\sigma}{\eta}\right)$, которому должны удовлетворять параметры краевой задачи, чтобы скорость $U$ обратилась в нуль в точке $Z_{1}$. Если наряду с $Z_{1}$ на интервале $(0,1)$ существуют еще нули полинома (15), то получим систему однородных линейных уравнений относительно тех же коэффициентов $\frac{g \beta h^{2}}{24 v}, \frac{\sigma}{\eta}$ и $\frac{a}{\delta}\left(\frac{g \beta h^{2}}{24 v}-\frac{\sigma}{\eta}\right)$. Известно, что такая система имеет нетривиальное решение только если ранг ее матрицы меньше числа неизвестных системы, т.е. если число нулей полинома (15) меньше трех. Другими словами, полином вида (15) может иметь не более двух корней внутри отрезка $[0,1]$.

Однако анализ свойств многочлена (9) с учетом условия $a>0$ показал, что скорость $U$ может иметь не более одной нулевой точки внутри слоя (рис. 3) при наложении потоков друг на друга. Именно эта точка является точкой стратификации поля скорости. Положение точки стратификации можно менять, варьируя значение длины проскальзывания $\alpha$, геометрических параметров слоя $(h, l)$ и физических параметров жидкости $(\beta, v$ и других).

При течении жидкости в ней возникает касательное напряжение

$$
\tau_{x z}=\eta \frac{\partial U}{\partial z}=\frac{\eta}{h} \frac{\partial U}{\partial Z}=A\left\{\frac{g \beta h^{2}}{6 v}\left[Z^{3}-3 Z+2\right]-\frac{\sigma}{\eta}\right\}
$$


которое также может один раз обратиться в нуль внутри слоя (рис. 4) при выполнении условия

$$
\left(g \beta h^{2} \eta-3 \vee \sigma\right) \sigma>0
$$

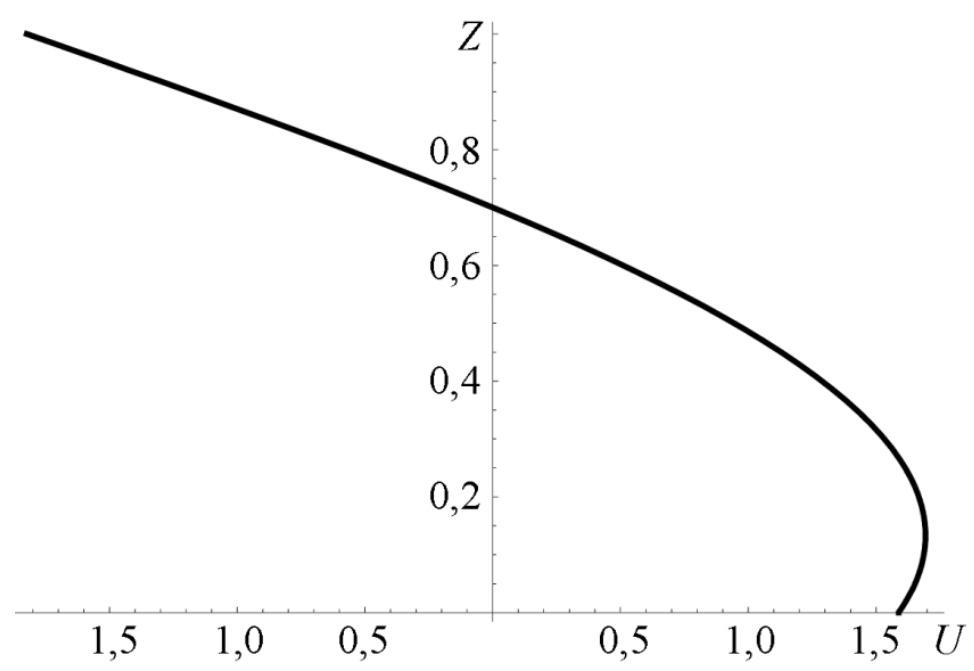

Рис. 3. Профиль скорости $U$

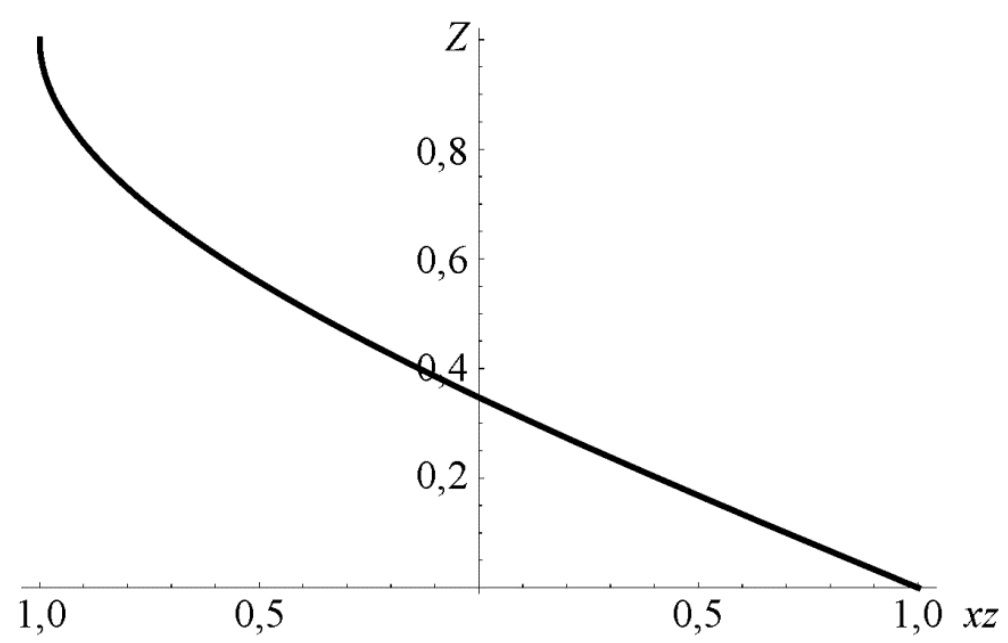

Рис. 4. Профиль касательного напряжения $\tau_{x z}$

Тип напряжения на верхней границе зависит от знака продольного градиента температуры $A$ и знака температурного коэффициента поверхностного натяжения $\sigma$. При этом величина касательного напряжения $\tau_{x z}(16)$ не зависит от безразмерной длины скольжения $a$, так как этот параметр входит в выражение для скорости $U$ (15) в виде аддитивного однородного слагаемого.

Заметим, что в случае прилипания жидкости или граничного условия для проскальзывания жидкости расход жидкости в рассматриваемом слое в общем случае будет ненулевым:

$$
Q=\int_{0}^{h} U(z) d z=h \int_{0}^{1} U(Z) d Z=A h^{2} \int_{0}^{1}\left\{\frac{g \beta h^{2}}{24 v}\left[Z^{4}-6 Z^{2}+8 Z\right]-\frac{\sigma}{\eta} Z+\frac{a}{\delta}\left(\frac{g \beta h^{2}}{24 v}-\frac{\sigma}{\eta}\right)\right\} d Z=
$$




$$
\begin{gathered}
=\left.A h^{2}\left[\frac{g \beta h^{2}}{24 v}\left(\frac{1}{5} Z^{5}-\frac{6}{3} Z^{3}+\frac{8}{2} Z^{2}\right)-\frac{\sigma}{2 \eta} Z^{2}+\frac{a}{\delta}\left(\frac{g \beta h^{2}}{24 v}-\frac{\sigma}{\eta}\right) Z\right]\right|_{0} ^{1}= \\
=A h^{2}\left[\frac{11 g \beta h^{2}}{120 v}-\frac{\sigma}{2 \eta}+\frac{a}{\delta}\left(\frac{g \beta h^{2}}{24 v}-\frac{\sigma}{\eta}\right)\right] .
\end{gathered}
$$

Заметим, что с учетом соотношения $v=\frac{\eta}{\rho}$, если справедливо равенство

$$
h^{2} \frac{11 h+5 \alpha}{h+2 \alpha}=\frac{60 \sigma}{g \beta \rho},
$$

связывающее геометрические параметры, характеризующие область течения и граничные условия, и физические параметры жидкости, то расход жидкости $Q$ для скольжения будет нулевым.

\section{3. Исследование скорости течения в случае идеального скольжения}

Условие Навье при бесконечном значении параметра $\alpha$ сводится к условию идеального скольжения. Если подставить $\alpha=\infty$ в приведенное выше точное решение (14), то получим, что все гидродинамические поля становятся бесконечными в любой точке исследуемого слоя. Все дело в том, что в предельном случае $\alpha=\infty$ меняется тип некоторых граничных условий, а именно, краевое условие третьего рода

$$
\left.\alpha \frac{\partial U}{\partial z}\right|_{z=0}=U(0)
$$

преобразуется к условию второго рода

$$
\left.\frac{\partial U}{\partial z}\right|_{z=0}=0,
$$

которое естественным образом следует из условия Навье (12) при $\alpha \rightarrow \infty$. В этом случае для получения частного решения необходимо апеллировать к общему решению (9) системы дифференциальных уравнений (8).

Подставим граничные условия (11) в точное решение (9) для продольного градиента температуры $T_{1}$ и получим:

$$
0=T_{1}(0)=C_{1} \cdot 0+C_{2} ; A=T_{1}(h)=C_{1} \cdot h+C_{2} .
$$

Из этой системы условий легко находим значения неизвестных констант: $C_{2}=0 ; C_{1}=A / h$. Далее используем граничные условия для градиента давления $P_{1}$ :

$$
0=P_{1}(h)=\frac{1}{2} g \beta h^{2} \cdot \frac{A}{h}+g \beta h \cdot 0+C_{3},
$$


откуда следует, что $C_{3}=-A g \beta h / 2$. Наконец, находим значения констант интегрирования в общем решении (9) для скорости $U$ :

$$
\begin{gathered}
0=\left.\frac{\partial U}{\partial z}\right|_{z=0}=\left.\left[\frac{\partial}{\partial z}\left(\frac{g \beta z^{4} C_{1}}{24 v}+\frac{g \beta z^{3} C_{2}}{6 v}+\frac{z^{2} C_{3}}{2 v}+z C_{4}+C_{5}\right)\right]\right|_{z=0}= \\
=\left.\left[\frac{4 g \beta z^{3}}{24 v} \cdot \frac{A}{h}+\frac{3 g \beta z^{3}}{6 v} \cdot 0-\frac{2 z^{2}}{2 v} \cdot \frac{A g \beta h}{2}+C_{4}\right]\right|_{z=0}=C_{4} .
\end{gathered}
$$

А значит, выражение для определения скорости $U$ примет вид:

$$
U(z)=\frac{A g \beta}{24 v h}\left[z^{4}-6 h^{2} z^{2}\right]+C_{5}=\frac{A g \beta h^{3}}{24 v}\left[Z^{4}-6 Z^{2}\right]+C_{5} .
$$

Для определения константы $C_{5}$ в выражении (17) необходимо использовать дополнительное условие. Мы будем брать условие нулевого расхода жидкости:

$$
\int_{0}^{1} U d Z=0,
$$

позволяющее асимптотически описывать течение в замкнутом слое. Учет условия (18) в решении (17) позволяет получить окончательный вид скорости $U$ :

$$
U=\frac{A g \beta h^{3}}{120 v} f_{3}(Z)=\frac{A g \beta h^{3}}{120 v}\left[5 Z^{4}-30 Z^{2}+9\right]
$$

График вспомогательной функции $f_{3}(Z)$ приведен на рис. 5.

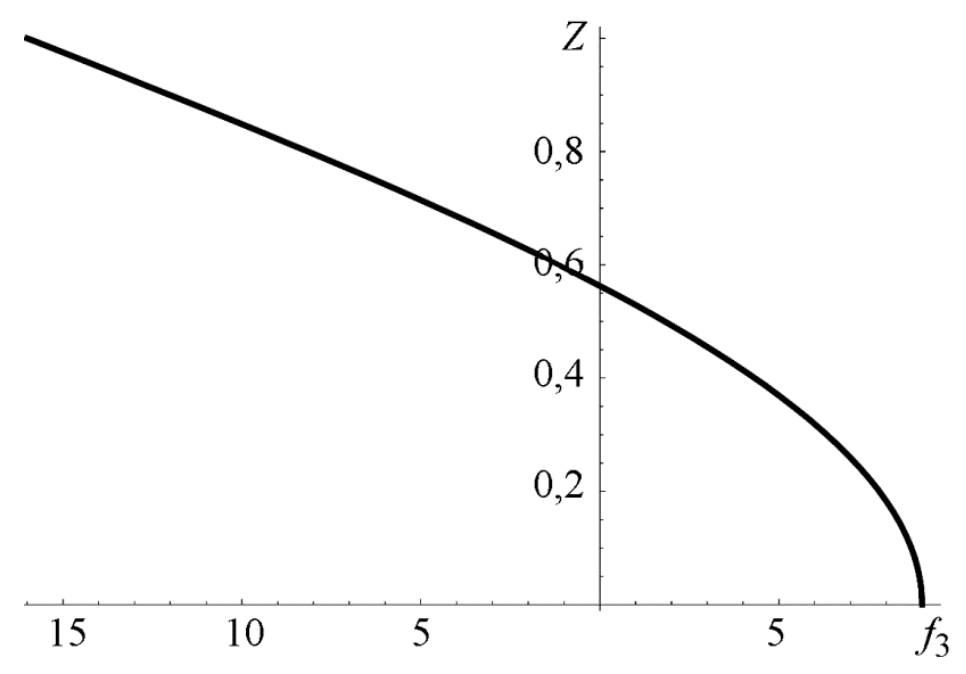

Рис. 5. График функции $f_{3}(Z)$ 
Рис. 5 иллюстрирует, что скорость $U$, определяемая выражением (19) имеет только одну нулевую точку, положение которой никак не зависит от значений параметров, задаваемых на границах слоя жидкости.

Однако следует помнить, что решение (19) должно удовлетворять еще и условию существования термокапиллярного эффекта:

$$
\left.\eta \frac{\partial U}{\partial z}\right|_{z=h}=-\sigma T_{1}(h)
$$

Это равносильно следующему условию:

$$
\begin{aligned}
-\sigma A=\left.\eta \frac{\partial U}{\partial z}\right|_{z=h}=\left.\eta\left[\frac{4 g \beta z^{3}}{24 v} \cdot \frac{A}{h}+\frac{3 g \beta z^{2}}{6 v} \cdot 0-\frac{2 z}{2 v} \cdot \frac{A g \beta h}{2}\right]\right|_{z=h}= \\
=\eta\left(\frac{A g \beta h^{2}}{6 v}-\frac{A g \beta h^{2}}{2 v}\right)=-\frac{A g \beta h^{2} \eta}{3 v} .
\end{aligned}
$$

Удовлетворение равенства

$$
g \beta h^{2} \eta-3 v \sigma=0
$$

обеспечивает непротиворечивость граничных условий, т.е. существование термокапиллярного эффекта на верхней границе при учете условия идеального скольжения на нижней границе исследуемого слоя жидкости.

Величина соответствующего касательного напряжения $\tau_{x z}$ в случае идеального скольжения может быть найдена по следующей формуле:

$$
\begin{gathered}
\tau_{x z}=\eta \frac{\partial U}{\partial z}=\frac{\eta}{h} \frac{\partial U}{\partial Z}=\frac{\eta}{h} \frac{\partial}{\partial z}\left(\frac{A g \beta h^{3}}{120 v}\left[5 Z^{4}-30 Z^{2}+9\right]\right)= \\
=\frac{A g \beta h^{2} \eta}{6 v} Z\left(Z^{2}-3\right) .
\end{gathered}
$$

Из выражения (21) легко видеть, что в отличие от случая $\alpha \in[0, \infty)$ напряжение $\tau_{x z}$ всюду в слое сохраняет знак. Тип напряжения (растягивающее или сжимающее) определяется знаком продольного градиента температуры $A$.

Посчитаем также, как это было проделано для случая выполнения условия проскальзывания, расход жидкости. Несложно убедиться, что в случае идеального скольжения он будет равен нулю:

$$
Q=h \int_{0}^{1} U(Z) d Z=\left.\frac{A g \beta h^{4}}{120 v}\left[Z^{5}-10 Z^{3}+9 Z\right]\right|_{0} ^{1}=\frac{A g \beta h^{4}}{120 v} \cdot[1-10+9]=0,
$$

что согласуется с условием (19), выбранным для определения необходимых констант в точном решении для поля скорости. 


\section{4. Заключение}

В статье получено новое точное решение, описывающее влияние термокапиллярного эффекта и условия проскальзывания на однонаправленное течение вязкой несжимаемой жидкости. Внимание в статье уделено анализу свойств поля скоростей течения. Показано, что полученное решение способно описывать возникновение стратификаций, при чем независимо от величины длины проскальзывания, скорость может иметь не более одной нулевой точки. Также было показано, что величина длины проскальзывания влияет на число нулевых точек компонент поля касательных напряжений.

\section{Литература}

1. Marangoni C. Sull espansione delle goccie di un liquido galleggiante sulla superficie di altro liquid. - Pavia : Tipografia dei fratelli Fusi, 1865.

2. Goldstein S. V. Modern Developments in Fluid Mechanics. - Oxford : Oxford Univ. Press, 1938.

3. Прандтль Л., Титьенс О. Гидро- и аэромеханика. Т.2. Движение жидкостей с трением и технические приложения. - М.-Л. : ОНТИ, 1935. - 283 с.

4. Борзенко Е. И., Шрагер Г. Р. Структура течения вязкопластичной жидкости при заполнении круглой трубы плоского канала // Вычисл. мех. сплошн. сред. - 2019. - Т. 12, вып. 2. - C. 129-136. - DOI: 10.7242/1999-6691/2019.12.2.11.

5. Neto C., Evans D., Bonaccurso E. Boundary slip in Newtonian liquids: a review of experimental studies // Reports on Progress in Physics. - 2005. - Vol. 68, no. 12 - P. 2859-2897. DOI: $10.1088 / 0034-4885 / 68 / 12 / \mathrm{R} 05$.

6. Течение вязкопластических нелинейных сред с пограничным проскальзыванием / В. В. Пеленко, В. А. Арет, Б. К. Гусев, Ф. В. Пеленко // Вестн. Красноярск. гос. аграрн. ун-та : межвуз. сб. науч. тр. - 2008. - № 2. - С. 54-57.

7. Navier C. L. M. H. M'emoire sur les lois du mouvement des fluides // M'em. Acad. Sci. Inst. de France. - 1823. - Vol. 2, no. 6. - P. 389-440.

8. Lauga E., Brenner M., Stone H. Microfluidics: The No-Slip Boundary Condition // Springer Handbook of Experimental Fluid Mechanics / ed by C. Tropea, A. L. Yarin, J. F. Foss. Berlin, Heidelberg : Springer Handbooks, 2007.

9. Hoffmann J., Johnson C. Computational Turbulent Incompressible Flow. - Heidelberg, Berlin : Springer-Verlag, 2007. - 397 p.

10. Борзенко Е. И., Дьякова О. А., Шрагер Г. Р. Исследование явления проскальзывания в случае течения вязкой жидкости в изогнутом канале // Вестн. Томск. гос. ун-та. Матем. и мех. - 2014. - Т. 2, вып. 28. - С. 35-44.

11. Привалова В. В., Просвиряков Е. Ю. Нелинейное изобарическое течение вязкой несжимаемой жидкости в тонком слое с проницаемыми границами // Вычисл. мех. сплошн. сред. - 2019. - Т. 12, вып. 2. - С. 230-242. - DOI: 10.7242/1999-6691/2019.12.2.20.

12. Гершуни Г. З., Жуховицкий Е. М. Конвективная неустойчивость несжимаемой жидкости. - М. : Наука, 1972. - 532 с.

13. Остроумов Г. А. Свободная конвекция в условиях внутренней задачи. - М. : Государственное изд-во технико-теоретической литературы, 1952.

14. Бирих Р. В. О термокапиллярной конвекции в горизонтальном слое жидкости / Р. В. Бирих // Прикладная механика и техническая физика. - 1966. - № 3. - С. 69-72.

15. Сидоров А. Ф. О двух классах решений уравнений механики жидкости и газа и их связи с теорией бегущих волн // ПМТФ. - 1989 - №. 2 - С. 34-40.

16. Mathematical Models of Convection / V. K. Andreev, Ya. A. Gaponenko, O. N. Goncharova, V. V. Pukhnachev. - Berlin-Boston : De Gryuter Publ., 2012. - xv+ + 417 p. 
17. Аристов С. Н., Просвиряков Е. Ю. Новый класс точных решений трехмерных уравнений термодиффузии // Теор. осн. хим. технолог. - 2016. - Т. 50, вып. 3. - Р. 294. DOI: $10.7868 / \mathrm{S} 0040357116030027$.

18. Aristov S. N., Frik P. G. Nonlinear effects of the Ekman layer on the dynamics of largescale eddies in shallow water // J. Appl. Mech. Tech. Phys. - 1991. - Vol. 32, no. 2. - P. 189-194. DOI: $10.1007 / \mathrm{BF} 00858033$.

19. Ингель Л. Х., Аристов С. Н. Класс точных решений нелинейных задач о термических циркуляциях, связанных с объемным тепловыделением в атмосфере // Тр. ин-та эксперим. метеорол. - 1991. - Вып. 27 (162). - С. 142-157.

20. Пухначев В. В. Нестационарные аналогии решения Бириха // Известия АлтГУ. - 2011. Вып. $69(1 / 2)$. - С. 62-69.

21. Аристов С. Н., Просвиряков Е. Ю. О слоистых течениях плоской свободной конвекции // Нелин. динам. - 2013. - Т. 9, вып. 4. - С. 651-657. - DOI: 10.20537/nd1304004.

22. Андреев В. К. Решения Бириха уравнений конвекции и некоторые его обобщения // Красноярск : Ин-т вычисл. мех. СО РАН, препринт №. 1-10, 2010. - 68 с.

23. Pukhnachev V. V. Group-theoretical methods in the convection problems // In: Application of Mathematics in Technical and Natural Sciences, edited by M. D. Todorov and C. I. Christov. 2011. - American Institute of Physics, CP 1404, Melwille, NY. - P. 31-42

24. Бурмашева Н. В., Просвиряков Е. Ю. Convective layered flows of a vertically whirling viscous incompressible fluid. Velocity field investigation // Вестн. Самарск. гос. техн. ун-та. Cер. Физ.-мат. науки. - 2019. - Т. 23, вып. 2. - С. 341-360. DOI: 10.14498/vsgtu1670

25. Аристов С. Н., Просвиряков Е. Ю., Спевак Л. Ф. Нестационарная конвекция БенараМарангони слоистых течений вязкой несжимаемой жидкости // Теор. основы хим. технолог. 2016. - T. 50, вып. 2. - C. 137-146. - DOI: 10.7868/S0040357116020019.

26. Burmasheva N. V., Prosviryakov E. Yu. Temperature field investigation in layered flows of a vertically swirling viscous incompressible fluid under two thermocapillar forces at a free boundary // Diagnostics, Resource and Mechanics of materials and structures. 2019. - Iss. 1. - P. 6-42. - DOI: 10.17804/2410-9908.2019.1.006-042. - URL: http://dreamjournal.org/DREAM_Issue_1_2019_Burmasheva_N.V._et_al._006_042.pdf

27. Горшков А. В., Просвиряков Е. Ю. Слоистая конвекция Бенара-Марангони при теплообмене по закону Ньютона-Рихмана // Комп. исслед. моделир. - 2016. - Т. 8, вып. 6. C. $927-940$.

28. Гончарова О. Н., Кабов О. А. Гравитационно-термокапиллярная конвекция жидкости в горизонтальном слое при спутном потоке газа // Доклады академии наук. - 2009. - Т. 54, вып. 5. - С. 183-188.

29. Бекежанова В. Б. Конвективная неустойчивость течения Марангони-Пуазейля при наличии продольного градиента температуры // Прикл. мех. техн. физ. - 2011. - Т. 52, вып. 1. C. $92-100$.

30. Гордеева В. Ю., Люшнин А. В. Влияние термокапиллярного эффекта на динамику и устойчивость движения испаряющейся тонкой пленки // Журн. техн. физ. - 2013. - Т. 83, вып. 3. - С. 41-47.

31. Актершев С. П. Термокапиллярный эффект и периодические структуры на поверхности нагреваемой пленки вязкой жидкости // Тр. инст. мех. УНЦ РАН. - 2007. - Т. 5, вып. 1. C. 79-84. - DOI: 10.21662/uim2007.1.005.

32. Справочник по теплообменникам : в 2 т. / пер. с англ. под ред. Б. С. Петухова, В. К. Шикова. - М. : Энергоатомиздат, 1987.

33. Aristov S. N., Prosviryakov E. Yu. Nonuniform convective Couette flow // Fluid Dynamics. 2016. - Vol. 51, no. 5. - P. 581-587. - DOI: 10.1134/S001546281605001X. 
34. Просвиряков Е. Ю. Новый класс точных решений уравнений Навье-Стокса со степенной зависимостью скоростей от двух пространственных координат // Теор. осн. хим. техн. 2019. - T. 53, вып. 1. - C. 112-120. - DOI: 10.1134/S0040357118060118.

35. Бурмашева Н. В., Просвиряков Е. Ю. Крупномасштабная слоистая стационарная конвекция вязкой несжимаемой жидкости под действием касательных напряжений на верхней границе. Исследование поля скоростей // Вестн. Самарск. гос. техн. ун-та. Сер. Физ.-мат. Науки. - 2017. - Т. 21, вып. 1. - C. 180-196. - DOI: 10.14498/vsgtu1527.

36. Knutova N. S., Shvarts K. G. A study of behavior and stability of an advective thermocapillary flow in a weakly rotating liquid layer under microgravity // Fluid Dyn. - 2015. - Vol. 50, no. 3. - P. 340-350. - DOI: 10.1134/S0015462815030047.

37. Couette M. Études sur le frottement des liquids // Ann. Chim. Phys. Ser. 6. - 1890. Vol. 21. - P. 433-510. 